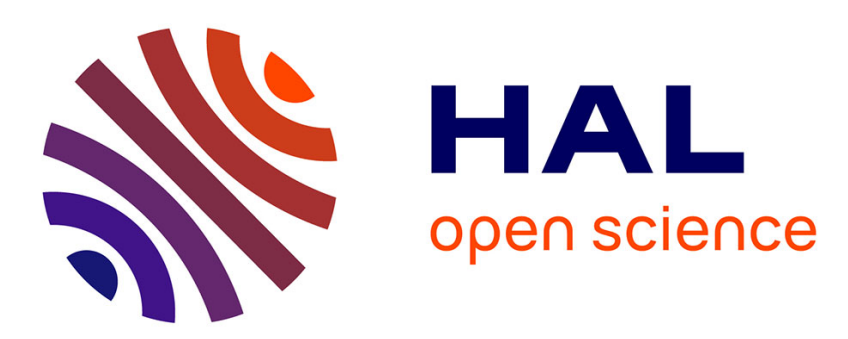

\title{
A Monte Carlo study of the magnetization reversal in DyFe2/YFe2 exchange-coupled superlattices
}

\author{
S. Djedai, Etienne Talbot, Pierre-Emmanuel Berche
}

\section{To cite this version:}

S. Djedai, Etienne Talbot, Pierre-Emmanuel Berche. A Monte Carlo study of the magnetization reversal in DyFe2/YFe2 exchange-coupled superlattices. Journal of Magnetism and Magnetic Materials, 2014, 368, pp.29 - 35. 10.1016/j.jmmm.2014.04.034 . hal-01633483

\section{HAL Id: hal-01633483 https://hal.science/hal-01633483}

Submitted on 16 Jan 2019

HAL is a multi-disciplinary open access archive for the deposit and dissemination of scientific research documents, whether they are published or not. The documents may come from teaching and research institutions in France or abroad, or from public or private research centers.
L'archive ouverte pluridisciplinaire HAL, est destinée au dépôt et à la diffusion de documents scientifiques de niveau recherche, publiés ou non, émanant des établissements d'enseignement et de recherche français ou étrangers, des laboratoires publics ou privés. 


\title{
A Monte Carlo study of the magnetization reversal in $\mathrm{DyFe}_{2} / \mathrm{YFe}_{2}$ exchange-coupled superlattices
}

\author{
S. Djedai ${ }^{\mathrm{a}, \mathrm{b}}$, E. Talbot ${ }^{\mathrm{a}}$, P.E. Berche ${ }^{\mathrm{a}, *}$ \\ ${ }^{a}$ Groupe de Physique des Matériaux, Université de Rouen, UMR 6634 CNRS, avenue de l'université - BP12, 76801 Saint Etienne du Rouvray, France \\ ${ }^{\mathrm{b}}$ Laboratoire de Physique Appliquée et Théorique, Université de Tebessa, BP 12002 Tebessa, Algeria
}

\begin{abstract}
A B S T R A C T
Monte Carlo simulations are used to perform an atomic scale modeling of the magnetic properties of epitaxial exchange-coupled $\mathrm{DyFe}_{2} / \mathrm{YFe}_{2}$ superlattices. These samples, extremely well-researched experimentally, are constituted by a hard ferrimagnet $\mathrm{DyFe}_{2}$ and a soft ferrimagnet $\mathrm{YFe}_{2}$ antiferromagnetically coupled. Depending on the layers' thickness and on the temperature, the field dependence of the magnetization depth profile is complex going from a unique giant ferromagnetic block to exchange spring behavior when the soft $\mathrm{YFe}_{2}$ layers reverse for positive bias fields. In some particular conditions of temperature and layers' thicknesses, it can even be easier to reverse the hard thin $\mathrm{DyFe}_{2}$ layers for positive fields, while the soft thick $\mathrm{YFe}_{2}$ layers keep their magnetization along the field. In this work, we reproduce by Monte Carlo simulations hysteresis loops for the net and compound-specific magnetizations at different temperatures, and assess the quality of the results by a direct comparison to experimental hysteresis loops.
\end{abstract}

\section{Introduction}

The study of exchange-coupled systems which combine two different magnetic materials that magnetically interact with each other at their interfaces has focused on an intense research activity for several years since they may exhibit interesting properties as spring magnet behavior and exchange bias effect [1]. Their potential application may concern permanent magnets [2] or data storage media $[3,4]$ for example. These applications need a good understanding of the magnetization switching in exchange-spring structures for which the multilayered films of Laves phase $\mathrm{REFe}_{2}$ $(\mathrm{RE}=$ rare earth $)$ grown by molecular beam epitaxy are very good system models [5]. DyFe $2 / \mathrm{YFe}_{2}$ superlattices are heterostructures that consist of hard $\mathrm{DyFe}_{2}$ and soft $\mathrm{YFe}_{2}$ ferrimagnetic layers. The hard magnetic phase tends to resist to magnetization reversal in high fields owing to its high anisotropy while the soft phase possesses large magnetization. There are three magnetic interactions that couple the moments of the rare earth and transition metal sites [6,7]. For DyFe 2 , these are $\mathrm{Fe}-\mathrm{Fe}$ interactions $J_{\mathrm{Fe}-\mathrm{Fe}}$, Dy-Fe interactions $J_{\text {Dy-Fe }}$ and Dy-Dy interactions $J_{\text {Dy-Dy }}$ with

$J_{\mathrm{Fe}-\mathrm{Fe}} \gg-J_{\mathrm{Dy}-\mathrm{Fe}}>J_{\text {Dy-Dy }}$.
In the case of $\mathrm{YFe}_{2}$ which is also ferrimagnetic but with a smaller induced moment essentially due to iron, its global magnetization is antiparallel to the one of $\mathrm{DyFe}_{2}$ as qualitatively shown in Fig. 1(a). The simplest scenario used to describe the magnetization reversal in such a hard/soft coupled system is usually the formation of exchange springs in the soft material (Fig. 1(b)), before the irreversible switch of the hard material [1].

However, some experimental studies have shown that this very simple description of the magnetization reversal is not valid in some circumstances of relatively high temperature or thin magnetic hard $\mathrm{DyFe}_{2}$ layers $[6,8,9]$. In that case, the magnetization reversal first affects the hard DyFe 2 layers whereas the $\mathrm{YFe}_{2}$ magnetization remains frozen in the applied field direction. This scenario requires a decrease of the hard layers' anisotropy energy due either to the high temperature or to the thinness of the $\mathrm{DyFe}_{2}$ layer.

These different experimental scenarios observed are the reason for which the numerical simulations at the atomic level may give a helpful tool to understand this wide variety of magnetic behaviors and are complementary to previous studies involving micromagnetic simulations [10].

\section{Model and numerical simulations}

We consider a face centered cubic (fcc) multilayered system made up of Fe, Dy and Y atoms. The direction of the uniaxial

\footnotetext{
* Corresponding author. Tel.: +332 329551 35; fax: +332 32955032 .

E-mail address: pierre.berche@univ-rouen.fr (P.E. Berche).
} 

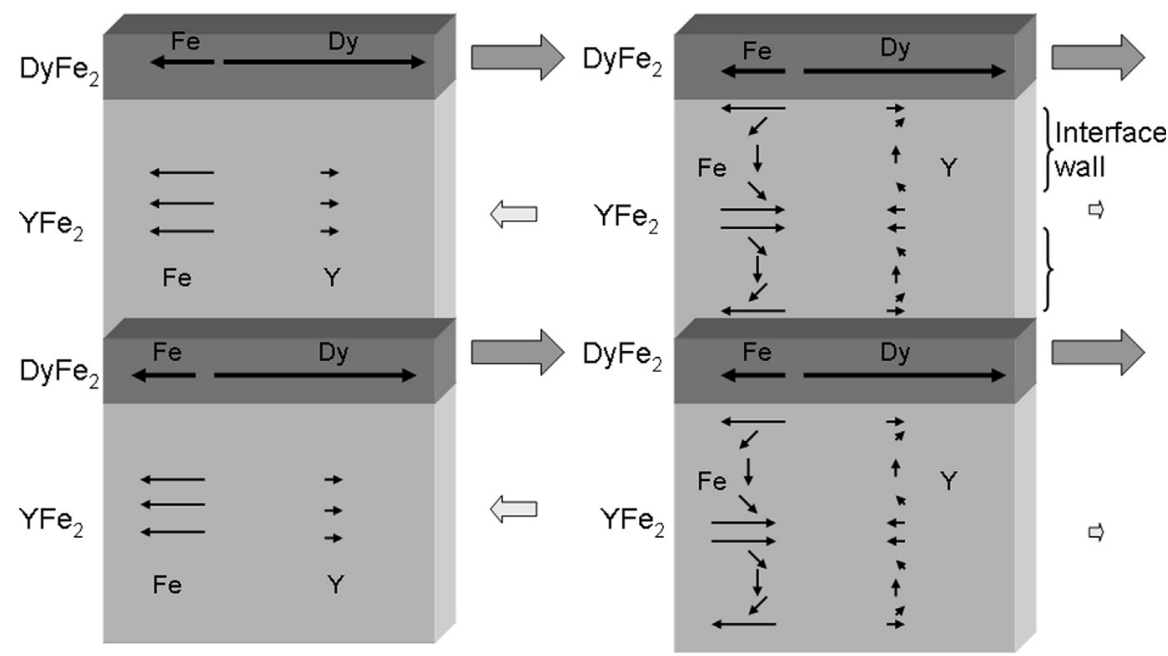

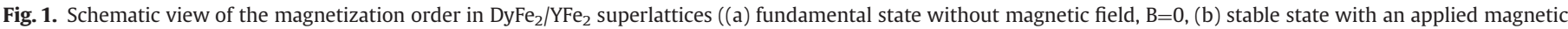
field $\mathrm{B} \neq 0$ ). The interface wall in the soft $\mathrm{YFe}_{2}$ layers is visible when the magnetization turns plane by plane. Two bilayers are shown in each case.

anisotropy has been identified, in experimental superlattices, as the $\left[\begin{array}{lll}1 & \overline{1} & 0\end{array}\right]$ in-plane direction whatever the temperature [11]. The SQUID measurements which the present work refers to have then been performed with an applied magnetic field along the same $\left[\begin{array}{lll}1 & \overline{1} & 0\end{array}\right]$ direction.

The Monte Carlo method in the canonical ensemble is used to investigate magnetization reversal in order to give a detailed microscopic description of the system. This method is wellknown to ensure a good convergence toward the thermodynamic equilibrium but it may in some circumstances not describe properly the magnetization reversal. So, we use here the algorithm proposed by Nowak et al. [12] with time step quantification. This technique allows us to get reliable Monte Carlo simulations with a reasonable number of Monte Carlo steps at low temperature. Moreover, unlike the standard Metropolis algorithm, this method prevents nonphysical spin flips by tunneling across the barrier. One Monte Carlo step (MCS) consists in examining all spins of the system once. At each temperature, 5000 MCSs were performed to reach the thermodynamic equilibrium, and afterwards the physical quantities were measured by averaging over the next 10000 MCSs. The model chosen is the classical Heisenberg model

$H=-\sum_{\langle i, j\rangle} J_{i j} \mathbf{S}_{\mathbf{i}} \cdot \mathbf{S}_{\mathbf{j}}-D\left(\right.$ Dy) $\sum_{i \in \text { Dy }}\left(\mathbf{S}_{\mathbf{i}} \cdot \mathbf{x}\right)^{2}-\mathbf{B} \cdot \sum_{i} \mathbf{m}_{\mathbf{i}}$,

where $\mathbf{S}_{\mathbf{i}}$ are the spin vectors at site $i$. The first term is the exchange energy over nearest neighbors of the same or different species, the second sum represents a uniaxial anisotropy which favors the so-called $x$-axis (corresponding in fact to the [1 $1 \overline{1} 0]$ direction) as the easy axis of the magnetization and concerns only the Dy atoms. Indeed, the strong magnetic anisotropy in the hard layer is due to the interaction of the crystal field with the RE ions [13]. Finally, the last sum corresponds to the Zeeman interaction with the magnetic field applied in the easy [ $\left[\begin{array}{lll}1 & 0\end{array}\right]$ direction.

The values of the magnetic parameters have been estimated from experimental data relative to the Curie temperatures of the multilayers and from the configuration of the magnetic moments of the different species in the fundamental state (Fig. 1(a)). The magnetization of the individual layers has been estimated in these superlattices at $0.9 \mu_{\mathrm{B}}$ /at. for the $\mathrm{YFe}_{2}$ layers and $1.65 \mu_{\mathrm{B}} /$ at. for the $\mathrm{DyFe}_{2}$ layers [5]. These values are in good agreement with those used by Bowden et al. for their magnetic modeling [14]. We observe that, due to the antiparallel orientation of the magnetization of the $\mathrm{DyFe}_{2}$ and $\mathrm{YFe}_{2}$ layers at low temperature, the ratio of the thicknesses which leads to a compensated magnetic state at $T=0$ and without any magnetic field is $e_{\mathrm{DyFe}_{2}} / e_{\mathrm{YFe}_{2}}=0.5$. Finally, the estimated values of the firstneighbors exchange interactions, already used in previous studies of Fe-Dy multilayers [15] except those concerning the Y atoms, are given in Table 1.

\section{Simulated versus experimental results}

As described in Section 1, different magnetization reversal processes can occur in the exchange-coupled superlattices depending on the layers' thicknesses and on the temperature. We present here a detailed description of these behaviors, as they are obtained experimentally and can be reproduced and interpreted by Monte Carlo simulations.

\subsection{Single-step reversal}

A Rigid magnetic Block ( $R B$ ) behavior when the switching is dominated by the hard DyFe 2 layer can be easily observed. The hysteresis loop is square and as for a single hard phase, there is no signature of magnetic walls in the $\mathrm{YFe}_{2}$ layers; the magnetization reversal is completely governed by the anisotropy in the DyFe $\mathrm{P}_{2}$ layers. The giant ferromagnetic superlattice behaves as unique block with a one step magnetization reversal, where the hard and soft magnetic layers switch as a unit for a coercive field which strongly decreases when the temperature increases (Fig. 2).

It has to be noticed that if the qualitative thermal evolution of the magnetization reversal is correctly reproduced by the numerical simulations, the quantitative value of the simulated coercive field is clearly overestimated. This effect is attributed to the magnetic parameters, especially the anisotropy constant, whose temperature dependence is not included in the Monte Carlo procedure. Then, our model underestimates probably the decrease of the energy barrier between the two magnetized opposite states. This scenario can also be observed when the soft layer $\mathrm{YFe}_{2}$ is thicker than the hard layer DyFe 2 but in the particular case of thin individual layer samples DyFe $2(1 \mathrm{~nm}) / \mathrm{YFe}_{2}(4 \mathrm{~nm})$ [6] such that the external magnetic field does not allow to break the ferrimagnetic configuration resulting from exchange. Indeed, in this case, 
the $\mathrm{YFe}_{2}$ layers are too thin to enable the development of magnetic walls. The large value of the coercive field has also to be noticed since at low temperature the ferrimagnetic configuration is maintained for a large negative field applied.

\subsection{Two-step reversal}

A two-step reversal can be observed with a Soft layers First (SF) switching and negative or positive coercivity. This scenario holds with an almost magnetically compensated behavior which is observed when the thickness of the soft layer is slightly larger than the hard layers one. In this superlattice, the $\mathrm{DyFe}_{2}$ and $\mathrm{YFe}_{2}$ net magnetizations can be almost equal and the ferrimagnetic arrangement stabilized after the first reversal step results then in approximately zero net moment (Fig. 3(1) and (2)).

From a general point of view, the measured hysteresis loops display a characteristic feature of magnetic exchange spring with the rise of an interface wall in the soft $\mathrm{YFe}_{2}$ layer where their cost is expected to be the smallest, as qualitatively shown in Fig. 1(b). We observe a very good qualitative agreement between the experimental and simulated curves describing the reversal of the magnetization but a relative discrepancy from a numerical point of view concerning the coercive field and the zero-field behavior. The reversal of the magnetization then occurs in two steps: firstly the rise of the interface wall, secondly the abrupt reversal of the hard DyFe ${ }_{2}$ layer. This scenario can even lead to the measurement

Table 1

Exchange interactions between nearest-neighbor atoms used in the simulations. The uniaxial anisotropy constant $D(\mathrm{Dy})$ due to the RE contribution is also given.

\begin{tabular}{llll}
\hline Exchange interactions & $J_{\mathrm{Fe}-\mathrm{Fe}} / k_{\mathrm{B}}$ & $J_{\mathrm{Fe}-\mathrm{Dy}} / k_{\mathrm{B}}$ & $J_{\mathrm{Dy}-\mathrm{Dy}} / k_{\mathrm{B}}$ \\
& $280 \mathrm{~K}$ & $-41.75 \mathrm{~K}$ & $6.5 \mathrm{~K}$ \\
& $J_{\mathrm{Fe}-\mathrm{Y}} / k_{\mathrm{B}}$ & $J_{\mathrm{Y}-\mathrm{Y}} / k_{\mathrm{B}}$ & $J_{\mathrm{Dy}-\mathrm{Y}} / k_{\mathrm{B}}$ \\
& $-41.75 \mathrm{~K}$ & $50 \mathrm{~K}$ & $10 \mathrm{~K}$ \\
Anisotropy constant & $D(\mathrm{Dy}) / k_{\mathrm{B}}=2 \mathrm{~K}$ & & \\
\hline
\end{tabular}

of a negative coercivity and a negative remanence when the magnetization of the whole superlattice is governed by the soft layer contribution, i.e. for a significative thicker $\mathrm{YFe}_{2}$ layer compared to the DyFe $\mathrm{F}_{2}$ layer (Fig. 3(3) and (4) where the soft $\mathrm{YFe}_{2}$ layer is 4 times thicker than the hard $\mathrm{DyFe}_{2}$ layer). But it has to be mentioned that this behavior is strongly dependent on the temperature since the $\mathrm{DyFe}_{2}$ magnetization decreases more rapidly in temperature than the $\mathrm{YFe}_{2}$ ones. Thus, the same samples at higher temperature ( $300 \mathrm{~K}$ for example) lead to a switching governed by the $\mathrm{YFe}_{2}$ soft layer which becomes magnetically dominant. This influence of the temperature on the reversal mechanism is investigated in the next section.

\subsection{Three-step reversal}

A Hard layer First (HF) reversal when the soft layer thickness is much larger than the hard layers one can also be observed although it corresponds to a less usual situation (Fig. 4). Several schematic spin configurations can be considered in function of the applied field (Fig. 4(2)).

The description of the magnetization reversal process is evidenced by the measurement of the magnetization of the hard DyFe 2 and soft $\mathrm{YFe}_{2}$ layers in the simulations (Fig. 4(3)).

This last situation is called Hard layer First (HF) switching since due to its small thickness, the anisotropy energy of the hard layer $\mathrm{DyFe}_{2}$ is not strong enough to stabilize it at the beginning of the reversal. Then we observe a first switching of the DyFe $\mathrm{F}_{2}$ layers for a large magnetic field applied followed by the reversal of the magnetization of the whole sample at a low field and finally the second switching of the DyFe 2 layers. Once again, the shape of the hysteresis loops is strongly dependent on the measurement temperature: when the temperature increases, the $\mathrm{DyFe}_{2}$ reversal is easier and the whole multilayer reverses for a smaller negative field.

In order to get a more accurate knowledge of the localization of the interface wall, in the two last cases (SF and HF), we have
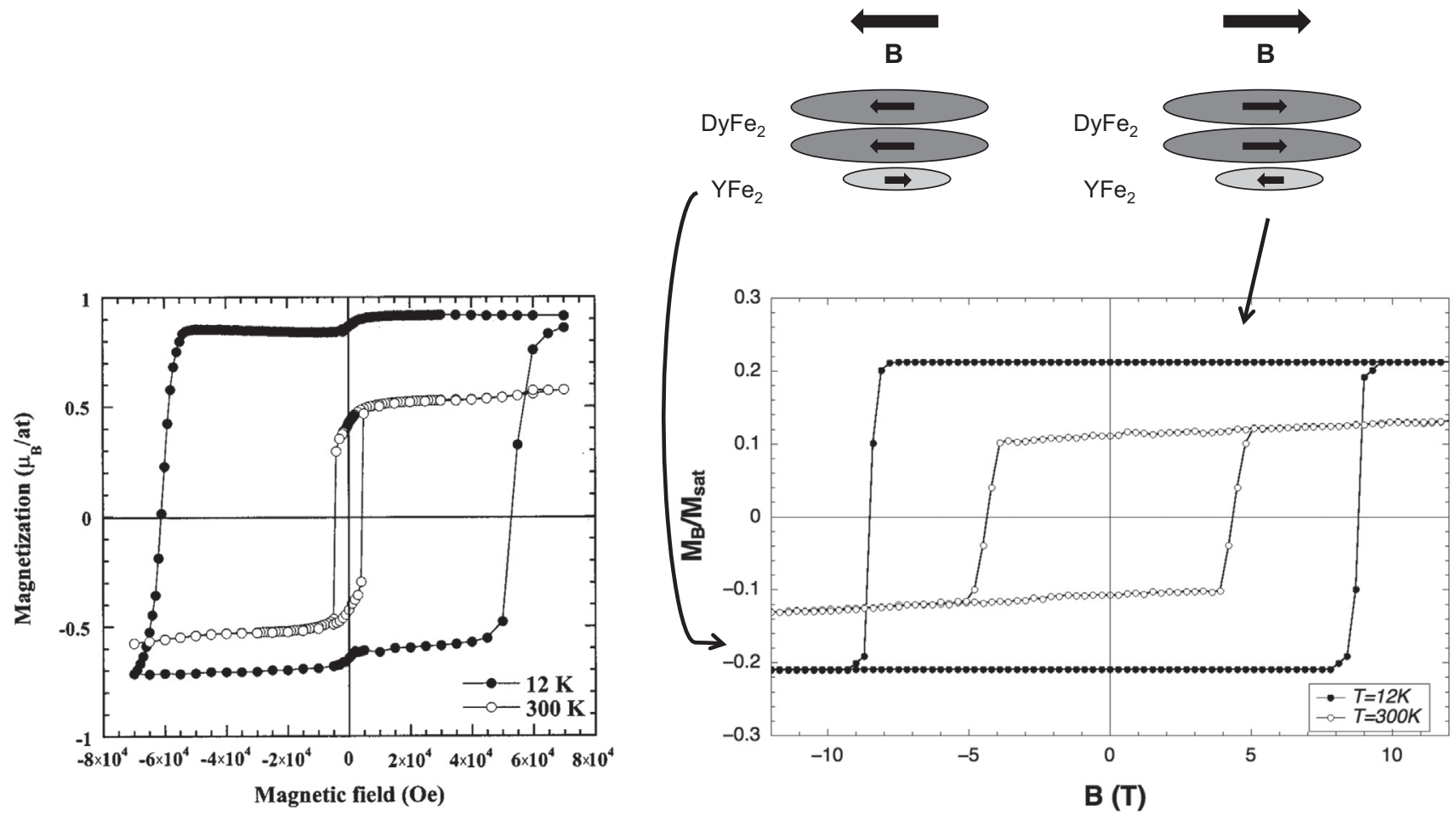

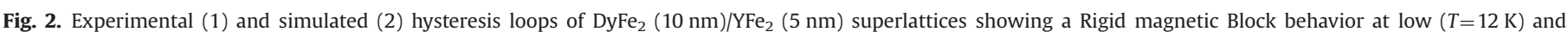
intermediate $(T=300 \mathrm{~K})$ temperatures [5]. 
(1)

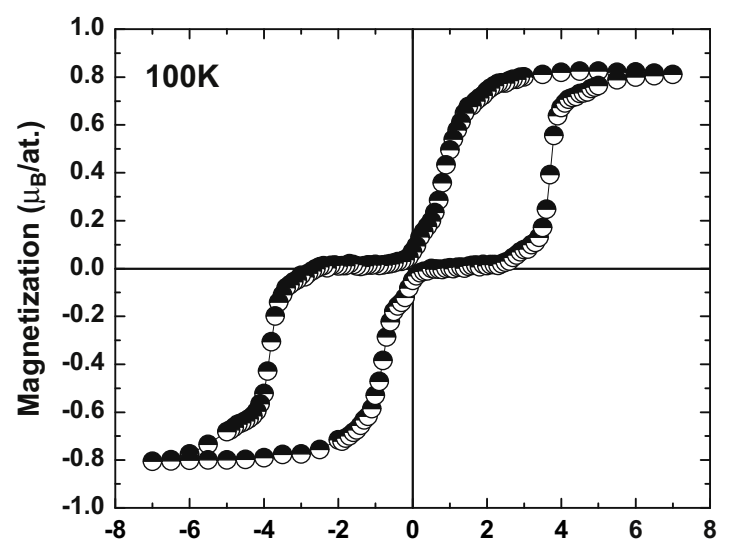

(2)

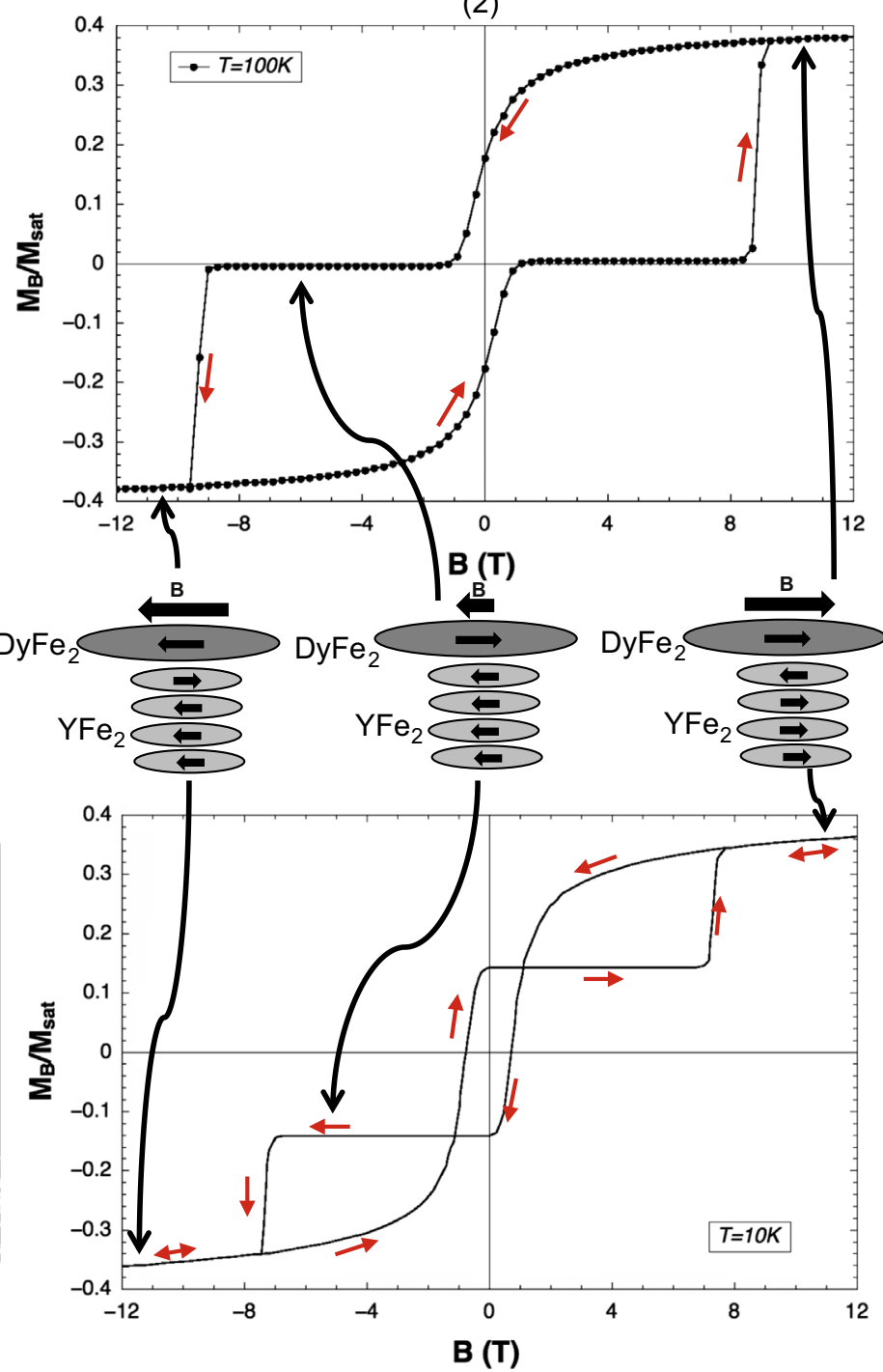

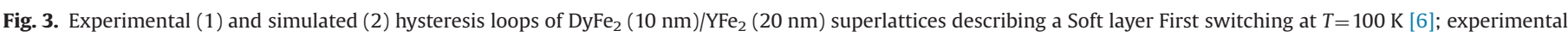

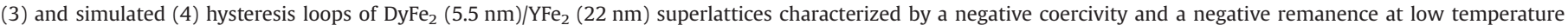
$(T=20 \mathrm{~K}[16]$ or $10 \mathrm{~K})$.

estimated, from the simulation data, the angle $\theta_{\mathrm{B}}$ between the directions of the magnetization of each atomic plane and the applied magnetic field (Fig. 5).

In the ferrimagnetic state, when the $\mathrm{DyFe}_{2}$ magnetization points up in the direction of the magnetic field whereas the $\mathrm{YFe}_{2}$ magnetization is opposite, the angle $\theta_{\mathrm{B}}$ is equal to 0 in the $\mathrm{DyFe}_{2}$ layer and $180^{\circ}$ in the $\mathrm{YFe}_{2}$ layer. The result of the simulated multilayer is shown in Fig. 5 for a superlattice DyFe $2(10 \mathrm{~nm}) / \mathrm{YFe}_{2}$ $(20 \mathrm{~nm})$ at low temperature $(T=10 \mathrm{~K})$.

We observe that the ferrimagnetic state is approximately reached only in the interface region and for a moderate applied magnetic field. Moreover, the interface wall, characterized by an angle different from 0 or $180^{\circ}$ is essentially located in the soft $\mathrm{YFe}_{2}$ layer with the magnetization of the core of the $\mathrm{YFe}_{2}$ layer which points up in the direction of the magnetic field. However, the magnetization of the $\mathrm{DyFe}_{2}$ atomic planes located near the interface is also disoriented relative to the field axis in order to satisfy the interface exchange couplings. These results are qualitatively in good agreement with previous experimental studies which show that for samples with rather thick individual thicknesses, the reversal of the magnetization is due to an exchange spring behavior: the soft $\mathrm{YFe}_{2}$ layers reverse for positive bias fields, then, the irreversible switch of the hard DyFe 2 layers follows [17-19].

\section{Influence of the temperature on the reversal process}

The study of the influence of the temperature shows that the reversal may evolve, for a same sample, from a Soft layer First (SF) mechanism below $100 \mathrm{~K}$ to a Hard layer First (HF) above $100 \mathrm{~K}$. In that case, the thermal energy may overcome the magnetic energy of the $\mathrm{DyFe}_{2}$ layers; thus, it becomes easier to reverse the hard $\mathrm{DyFe}_{2}$ layers for positive fields while the dominant magnetization of the soft $\mathrm{YFe}_{2}$ layers remains aligned along the field. This result has been numerically evidenced with the DyFe $2.5 \mathrm{~nm}) / \mathrm{YFe}_{2}$ $(22 \mathrm{~nm})$ multilayers for which the typical hysteresis loops are shown at $T=10 \mathrm{~K}$ and $T=300 \mathrm{~K}$ (Fig. 6(1) and (2)) and the coercive field $B_{c}$ has been measured in function of the temperature (Fig. 6(3)).

The same typical results have been observed experimentally with the same layers' thicknesses [6]. The magnetization reversal has then been characterized by the measurement of the magnetic 
(1)

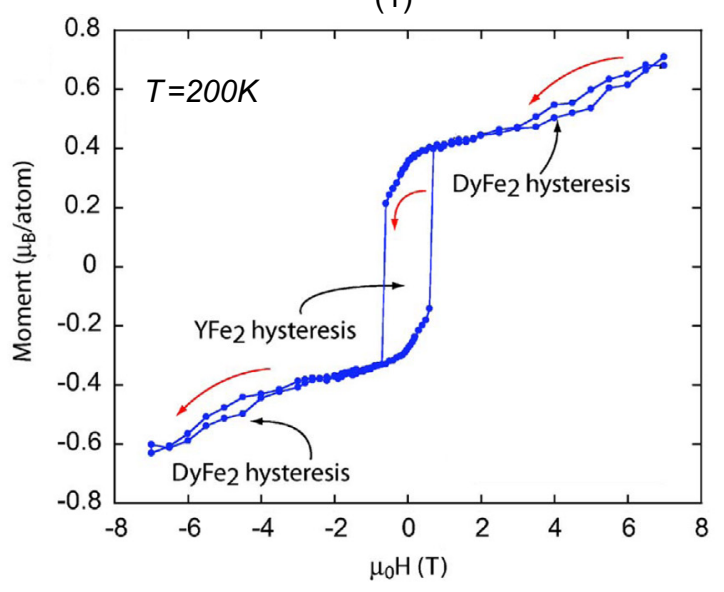

(3)

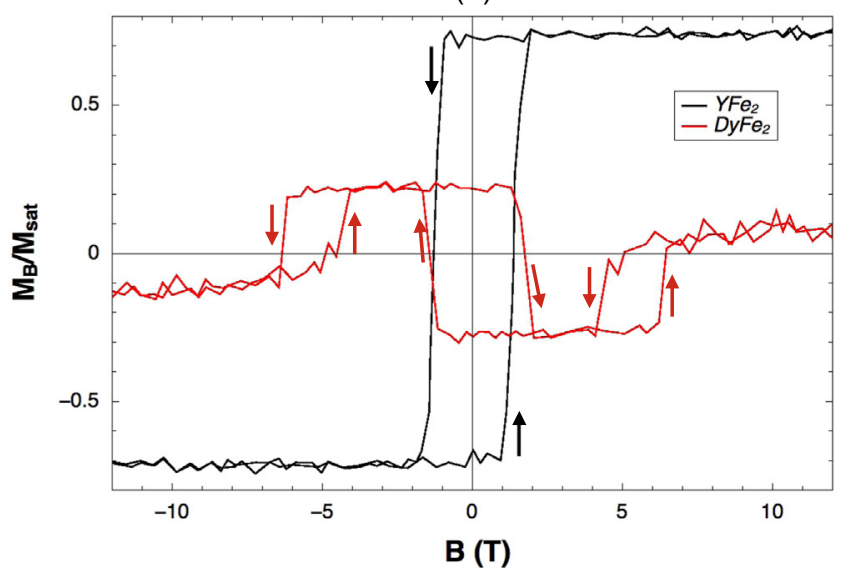

(2)

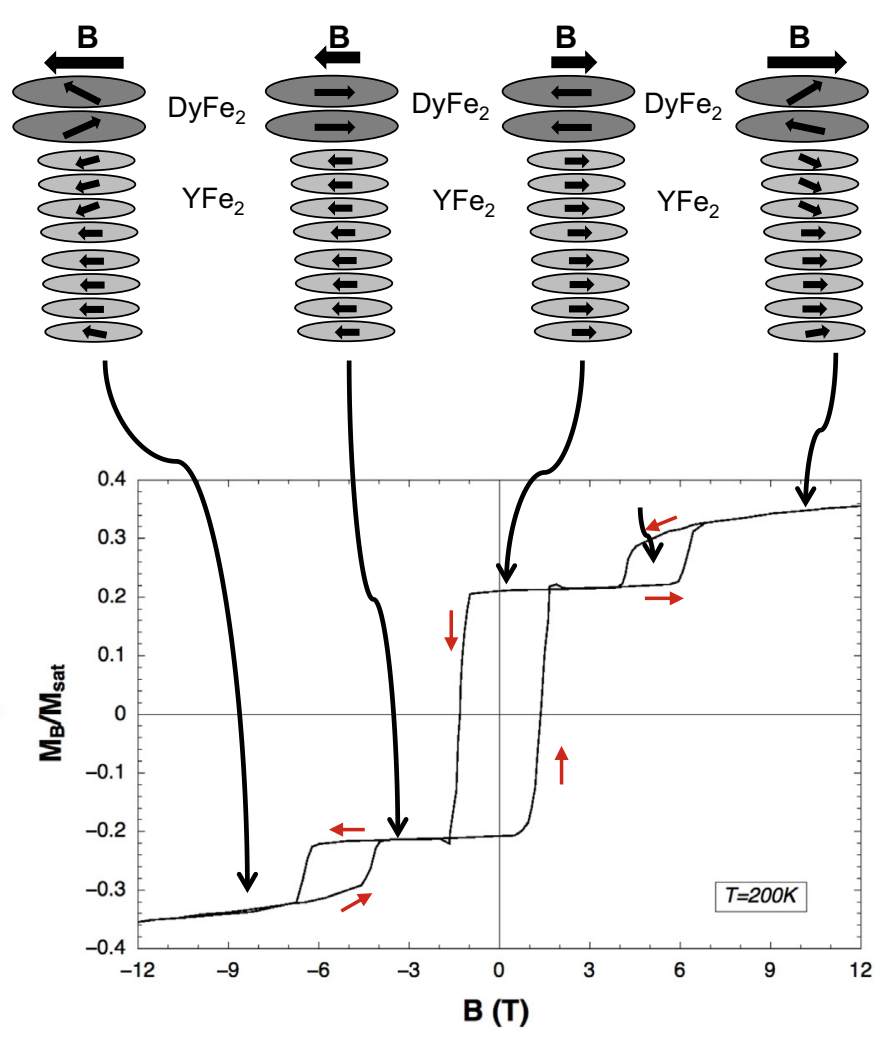

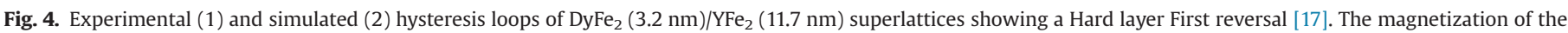
hard $\mathrm{DyFe}_{2}$ and soft $\mathrm{YFe}_{2}$ layers by Monte Carlo simulations which allows us to describe the reversal process of the DyFe $\mathrm{I}_{2}$ layers in three steps is also shown (3).

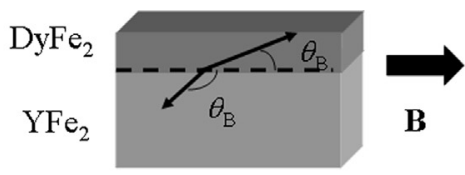

a

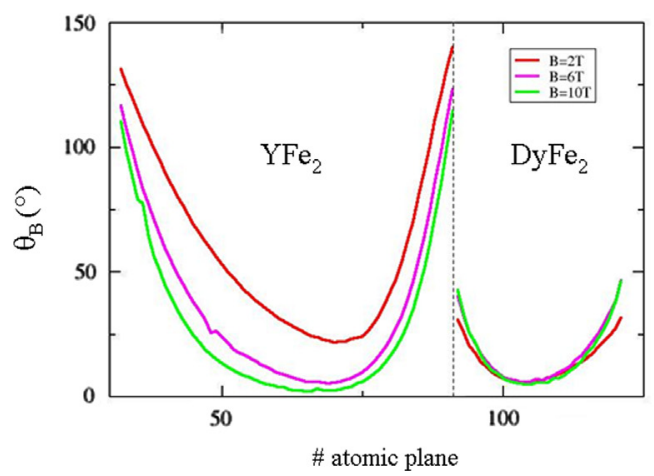

$\mathrm{YFe}_{2}$

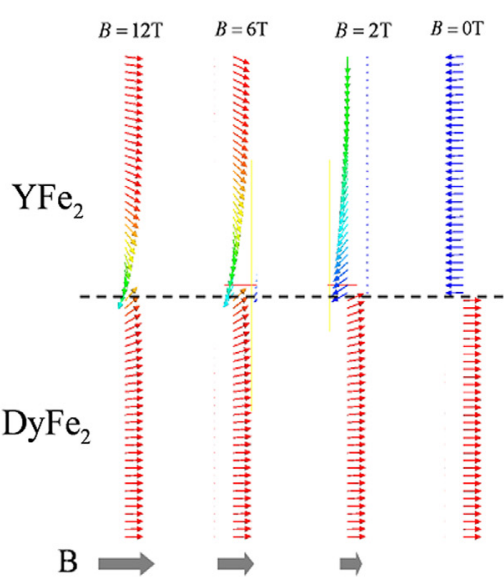

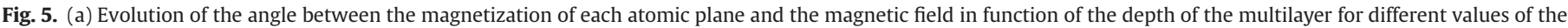

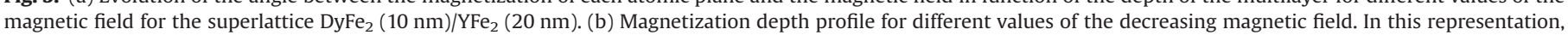

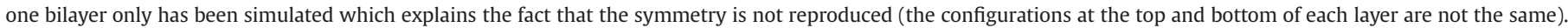

field $\left(H_{Y}\right)$ for which the $\mathrm{YFe}_{2}$ magnetization reverses. This quantity, calculated in function of the temperature, allows us to determine 3 different temperature ranges: a "SF" region below $150 \mathrm{~K}$, an "HF" region above $200 \mathrm{~K}$ and an "intermediate" case between 150 and $200 \mathrm{~K}$ where both compounds are involved in the first step of reversal. 
(1)

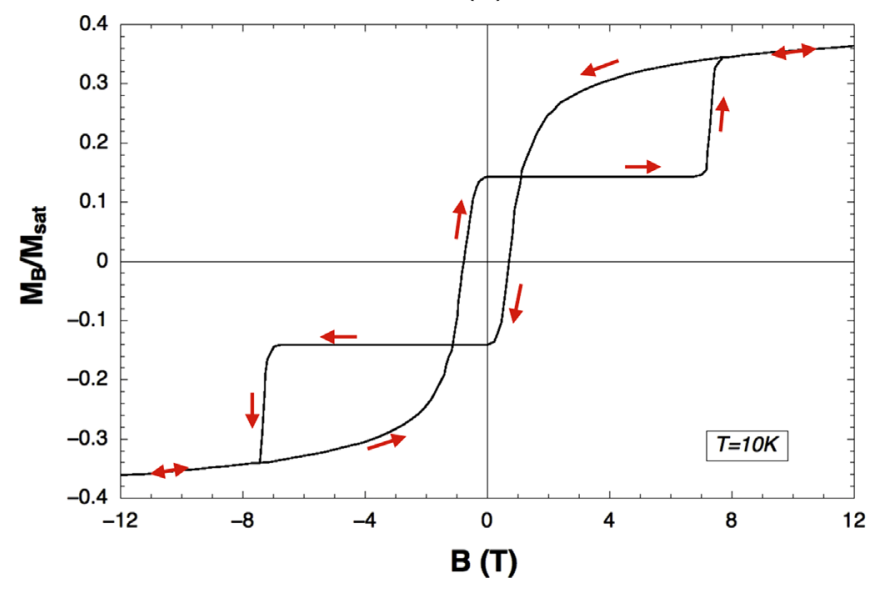

(2)

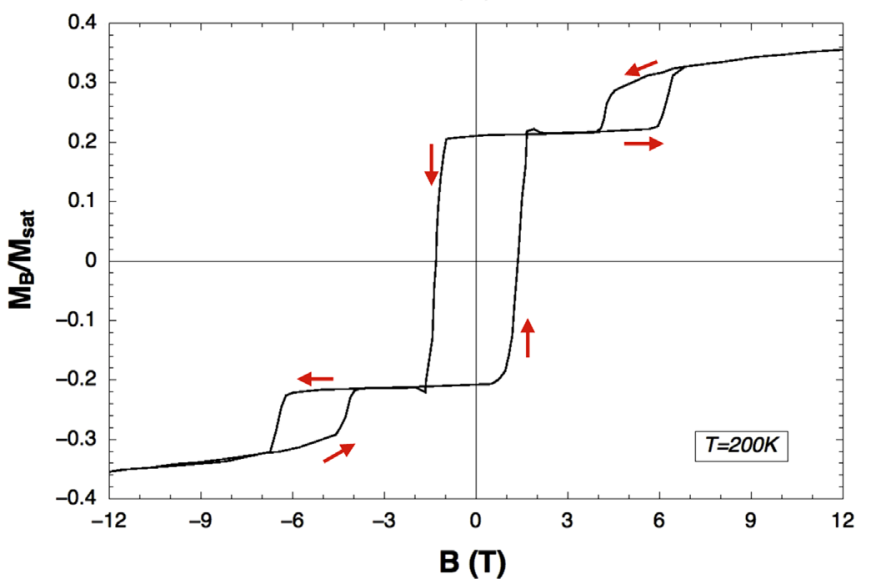

(3)

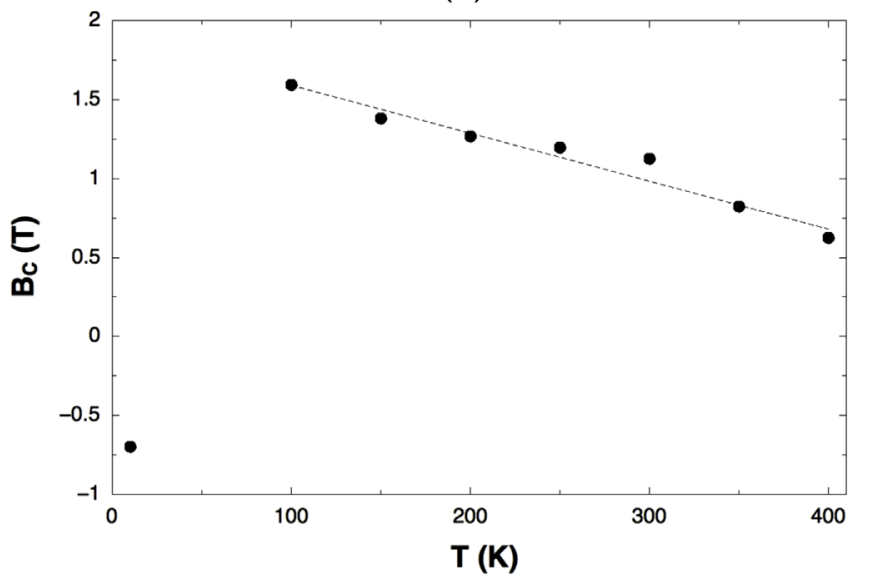

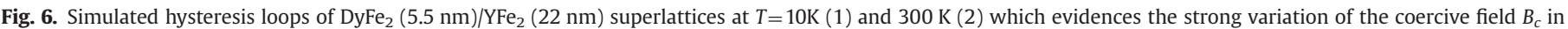
function of the temperature (3).

It has also to be mentioned that micromagnetic simulations have been performed [10] which qualitatively reproduce the existence of two temperature regimes with the hard $\mathrm{DyFe}_{2}$ layers switching at finite high temperature.

\section{Conclusion}

In this paper, it was shown that the Monte Carlo method, adapted to include magnetic exchange springs, can be used to provide a good semi-quantitative interpretation of the switching of the magnetization in DyFe $2 / \mathrm{YFe}_{2}$ multilayers. In particular, we have been able to reproduce hysteresis loops with characteristics matching those of experimental works at different temperatures. A strong temperature dependence of the magnetization reversal plays a key role for the reversal processes, and the interplay with exchange and Zeeman energy is understood on a qualitative level. The variation of the coercive field in function of the temperature, which depends not only on the soft/hard layers thicknesses ratio but also on the individual thicknesses, is due to the thermal change of the magnetic dominant configuration. The results of this study are in qualitative very good agreement with a great number of experimental works.

\section{Acknowledgments}

The simulations were performed at the Centre de Ressources Informatiques de Haute Normandie (CRIHAN) under the project No. 2008001. Moreover, the authors are indebted to Dr. Catherine Dufour (deceased) and Dr. Karine Dumesnil from the Institut Jean Lamour of Nancy University for valuable discussions on the experimental results.

\section{References}

[1] E.E. Fullerton, J.S. Jiang, M. Grimsditch, C.H. Sowers, S.D. Bader, Phys. Rev. B 58 (1998) 12193.

[2] R. Skomski, J.M.D. Coey, Phys. Rev. B 48 (1993) 15812.

[3] D. Suess, T. Schrefl, S. Fahler, M. Kirschner, G. Hrkac, F. Dorfbauer, J. Fidler, Appl. Phys. Lett. B 87 (2005) 012504.

[4] R.H. Victora, X. Shen, IEEE Trans. Mag. 41 (2005) 537.

[5] K. Dumesnil, M. Dutheil, C. Dufour, Ph. Mangin, Phys. Rev. B 62 (2000) 1136.

[6] K. Dumesnil, S. Fernandez, A. Avisou, C. Dufour, A. Rogalev, F. Wilhelm, E. Snoeck, Eur. Phys. J. B 72 (2009) 159.

[7] M.J. Bentall, R.A. Cowley, W.J.L. Buyers, Z. Tun, W. Lohstroh, R.C.C. Ward, M. R. Wells, J. Phys.: Condens. Matter 15 (2003) 4301.

[8] O. Hellwig, T.L. Kirk, J.B. Kortright, A. Berger, E.E. Fullerton, Nat. Mater. 2 (2003) 112.

[9] G.B.G. Stenning, A.R. Buckingham, G.J. Bowden, R.C.C. Ward, G. van der Laan, L. R. Shelford, F. Maccherozzi, S.S. Dhesi, P.A.J. de Groot, Phys. Rev. B 84 (2011) 104428. 
[10] J.P. Zimmermann, G. Bordignon, R.P. Boardman, J. Fischbacher, H. Fangohr K.N. Martin, G.J. Bowden, A.A. Zhukov, P.A.J. de Groot, J. Appl. Phys. 99 (2006 08B904.

[11] A. Mougin, C. Dufour, K. Dumesnil, Ph. Mangin, Phys. Rev. B 62 (2000) 9517.

12] U. Nowak, R.W. Chantrell, E.C. Kennedy, Phys. Rev. Lett. 84 (2000) 163.

[13] G.J. Bowden, D.St.P. Bunbury, A.P. Guimares, R.E. Snyder, J. Phys. C: Solid State Phys. 1 (1968) 1376

[14] G.J. Bowden, J.M.L. Beaujour, A.A. Zhukov, B.D. Rainford, P.A.J. De Groot, R.C.C. Ward, M.R. Wells, J. Appl. Phys. 93 (2003) 6480.
[15] E. Talbot, D. Ledue, P.E. Berche, J. Appl. Phys. 106 (2009) 023901.

[16] J.M.L. Beaujour, S.N. Gordeev, G.J. Bowden, P.A.J. De Groot, B.D. Rainford, R.C.C. Ward, M.R. Wells, Appl. Phys, Lett. 78 (2001) 964

[17] M.R. Fitzsimmons, S. Park, K. Dumesnil, C. Dufour, R. Pynn, J.A. Borchers, J.J. Rhyne, Ph. Mangin, Phys. Rev. B 73 (2006) 134413.

[18] K. Dumesnil, C. Dufour, Ph. Mangin, A. Rogalev, Phys. Rev. B 65 (2002) 094401.

[19] M. Sawiki, G.J. Bowden, P.A.J. De Groot, B.D. Rainford, J.M.L. Beaujour, R.C.C. Ward, M.R. Wells, Phys. Rev. B 62 (2000) 5817. 